University of Nebraska - Lincoln

DigitalCommons@University of Nebraska - Lincoln

USDA National Wildlife Research Center - Staff Publications
U.S. Department of Agriculture: Animal and Plant Health Inspection Service

2012

\title{
Identification and Analysis of the First 2009 Pandemic H1N1 Influenza Virus from U.S. Feral Swine
}

A. Clavijo

Texas Veterinary Medical Diagnostic Laboratory, aclavijo@paho.org

Amir Nikooienejad

Texas AgriLife Research, Nikooienejad@tamu.edu

M. S. Esfahani

Texas AgriLife Research, m.shahrokh@tamu.edu

Richard P. Metz

Texas AgriLife Research, rmetz@tamu.edu

Scott Schwartz

Texas AgriLife Research, sschwartz@ag.tamu.edu

See next page for additional authors

Follow this and additional works at: https://digitalcommons.unl.edu/icwdm_usdanwrc

Part of the Life Sciences Commons

Clavijo, A.; Nikooienejad, Amir; Esfahani, M. S.; Metz, Richard P.; Schwartz, Scott; Atashpaz-Gargari, Esmaeli; Deliberto, Thomas J.; Lutman, M. W.; Pedersen, Kerri; Bazan, L. R.; Koster, L. G.; Jenkins-Moore, M.; Swenson, S. L.; Zhang, M.; Beckham, T.; Johnson, Charles D.; and Bounpheng, Mohammad, "Identification and Analysis of the First 2009 Pandemic H1N1 Influenza Virus from U.S. Feral Swine" (2012). USDA National Wildlife Research Center - Staff Publications. 1116.

https://digitalcommons.unl.edu/icwdm_usdanwrc/1116

This Article is brought to you for free and open access by the U.S. Department of Agriculture: Animal and Plant Health Inspection Service at DigitalCommons@University of Nebraska - Lincoln. It has been accepted for inclusion in USDA National Wildlife Research Center - Staff Publications by an authorized administrator of DigitalCommons@University of Nebraska - Lincoln. 


\section{Authors}

A. Clavijo, Amir Nikooienejad, M. S. Esfahani, Richard P. Metz, Scott Schwartz, Esmaeli Atashpaz-Gargari, Thomas J. Deliberto, M. W. Lutman, Kerri Pedersen, L. R. Bazan, L. G. Koster, M. Jenkins-Moore, S. L. Swenson, M. Zhang, T. Beckham, Charles D. Johnson, and Mohammad Bounpheng 
ORIGINAL ARTICLE

\title{
Identification and Analysis of the First 2009 Pandemic H1N1 Influenza Virus from U.S. Feral Swine
}

\author{
A. Clavijo ${ }^{1}$, A. Nikooienejad ${ }^{2}$, M. S. Esfahani ${ }^{2}$, R. P. Metz ${ }^{2}$, S. Schwartz ${ }^{2}$, E. Atashpaz-Gargari ${ }^{2}$
} T. J. Deliberto ${ }^{3}$, M. W. Lutman ${ }^{3}$, K. Pedersen ${ }^{3}$, L. R. Bazan ${ }^{4}$, L. G. Koster ${ }^{5}$, M. Jenkins-Moore ${ }^{5}$,

S. L. Swenson ${ }^{5}$, M. Zhang ${ }^{1}$, T. Beckham ${ }^{1}$, C. D. Johnson ${ }^{2}$ and M. Bounpheng ${ }^{1}$

1 Texas Veterinary Medical Diagnostic Laboratory, College Station, TX, USA

2 Texas AgriLife Research, College Station, TX, USA

3 United States Department of Agriculture, Fort Collins, CO, USA

${ }^{4}$ United States Department of Agriculture, San Diego, TX, USA

${ }^{5}$ National Veterinary Services Laboratories, Ames, IA, USA

\section{Impacts}

- For the first time, a pandemic H1N1 virus was identified from feral swine in the United States.

- Next generation sequencing can be used to determine the pathogen of interest for differential diagnosis directly from biological diagnostics samples.

- Epidemiological studies are required to understand the role of feral swine as reservoir and transmitting host to other susceptible species and ultimately to human population.

\section{Keywords:}

Pandemic influenza; pH1N1; next generation sequencing; feral swine

\section{Correspondence:}

A. Clavijo. Pan American Health Organization, Av. Presidente Kennedy, 7778, Duque de Caxias CEP, 25040-004, Rio de Janeiro, Brazil. Tel.: +55 213661 9080; Fax: +55 213661 9001; E-mail: aclavijo@paho.org

Received for publication March 20, 2012

doi: 10.1111/zph.12006

\section{Summary}

The first case of pandemic H1N1 influenza (pH1N1) virus in feral swine in the United States was identified in Texas through the United States Department of Agriculture (USDA) Wildlife Services' surveillance program. Two samples were identified as pandemic influenza by reverse transcriptase quantitative PCR (RT-qPCR). Full-genome Sanger sequencing of all eight influenza segments was performed. In addition, Illumina deep sequencing of the original diagnostic samples and their respective virus isolation cultures were performed to assess the feasibility of using an unbiased whole-genome linear target amplification method and multiple sample sequencing in a single Illumina GAIIx lane. Identical sequences were obtained using both techniques. Phylogenetic analysis indicated that all gene segments belonged to the pH1N1 (2009) lineage. In conclusion, we have identified the first $\mathrm{pH} 1 \mathrm{~N} 1$ isolate in feral swine in the United States and have demonstrated the use of an easy unbiased linear amplification method for deep sequencing of multiple samples.

\section{Introduction}

The 2009 pandemic influenza A H1N1 (pH1N1) virus was detected in humans in the United States in early April 2009 (Dawood et al., 2009); the virus was subsequently reported in more than 200 countries and resulted in over 18000 deaths by August 2010 (World Health Organization, Global Alert and Response (GAR), 2012). Shortly after human infections were detected, pH1N1 infections in domestic pigs were identified in
Canada in early May 2009 (Forgie et al., 2011). Since then, pH1N1 infections, presumably transmitted from humans to domestic pigs, have been reported in more than 20 countries (NCBI Influenza virus sequence database, 2012, Njabo et al., 2012). Experimental and epidemiological data suggest that $\mathrm{pH} 1 \mathrm{~N} 1$ can be transmitted between pigs, as well as from pigs to humans, and vice versa (Nfon et al., 2011).

Feral swine are considered an invasive species in the United States. Their current population is estimated at 
roughly five million pigs in 38 states (Wyckoff et al., 2009), but their prolific and adaptable nature have resulted in a feral swine population that expands into new states and counties every year. Feral swine cause over $\$ 1$ billion in damage to the U.S. agricultural industry each year (Pimental, 2007) and pose a risk to livestock and native wildlife by reintroducing endemic diseases into areas previously considered disease-free (Saliki et al., 1998). As the populations and ranges of feral swine rapidly expand across the United States, the economic and biological impacts of feral swine will require greater scrutiny.

The USDA Wildlife Services' (WS) National Wildlife Disease Program (NWDP) conducts comprehensive surveillance for many diseases (i.e., classical swine fever (Swafford et al., 2009), pseudorabies, swine brucellosis (Pedersen et al., 2012), swine influenza, toxoplasmosis, trichinellosis and hepatitis E virus) found in feral swine. Wildlife disease biologists opportunistically collect tissue and blood samples from feral swine, which are then screened for more than 10 different pathogens. In November 2010, the NWDP initiated a swine influenza virus (SIV) surveillance program of feral swine to understand the diversity and distribution of SIV including pH1N1. This surveillance is meant to complement the efforts currently employed for domestic swine populations outlined in the Veterinary Services National Surveillance Plan for SIV in Pigs (Animal and Plant Inspection Services, 2012). Here, we report the identification, sequencing and phylogenetic analysis of the first 2009 pH1N1 isolate from feral swine in the United States.

\section{Material and Methods}

\section{Samples}

On 15 June 2011, WS employees in Motley County, Texas, collected samples from two feral swine that were shot in a cotton field. Nasal swabs (NS) were obtained from both nostrils of each animal and placed into cryovials containing $2 \mathrm{ml}$ of brain-heart infusion (BHI) medium. These samples (identified as NS10253 and NS10254) were stored under refrigeration and shipped with ice packs to the Texas Veterinary Medical Diagnostic Laboratory for RT-qPCR testing. Blood samples were also collected via cardiac puncture, and the serum was shipped to the NWDP in Fort Collins, Colorado, where influenza A serology was conducted (Ciacci-Zanella et al., 2010).

\section{Isolation of virus and the purification and detection of Viral RNA}

Virus was isolated using Madin-Darby canine kidney cells according to previously described methods (Clavijo et al.,
2002). Virus isolation culture (VIC) samples were identified as VIC10253 and VIC10254.

Purification of viral RNA and RT-qPCR of all samples were performed using USDA validated procedures. Briefly, viral RNA was purified from a $50-\mu$ l sample using a MagMAX ${ }^{\mathrm{TM}}-96$ AI/ND Viral RNA Isolation Kit (Life Technologies, Grand Island, NY, USA) and the KingFisher 96 Magnetic Particle Processor (Thermo Fisher Scientific, Waltham, MA, USA). Purified RNA was eluted in $50 \mu \mathrm{l}$ of elution buffer, and $8 \mu \mathrm{l}$ of the purified RNA was used for RT-qPCR using assay-specific primers and probes and the AgPath-ID ${ }^{\mathrm{TM}}$ One-Step RT-PCR Reagents (Life Technologies).

\section{Sanger sequencing}

Viral RNA was purified from virus cultures using an Ambion MagMAX ${ }^{\mathrm{TM}}$-96 Viral RNA Isolation Kit (Life Technologies). Gene segments were amplified in overlapping fragments using One-Step RT-PCR Reagents (Qiagen, Valencia, CA, USA) and specific primers (Chan et al., 2006). Additional primers were designed in-house to cover the entire genome and facilitate sequencing. The PCR amplicons were purified using a QIAquick PCR Purification Kit (Qiagen) and were then used for sequencing using a BigDye Terminator v3.1 Cycle Sequencing Kit (Life Technologies) according to the manufacturer's instructions. Sequencing reactions were performed using the Applied Biosystems 3730xl Genetic Analyzer system (Life Technologies). The sequences were then assembled and edited with the sequencher v4.9 software (Gene Codes Corp., Ann Arbor, MI, USA).

\section{Illumina GAIIx sequencing}

The SIV-positive nasal swab samples were diluted $1: 10$ and $1: 100$ in a negative nasal swab suspension pool (NSP) to assess the feasibility of direct biological sample next generation sequencing (NGS) using the Illumina GAIIx platform. Viral RNA samples from nasal swab samples were treated with the Ribo-Zero ${ }^{\mathrm{TM}}$ rRNA Removal Kit Low Input (Epicentre Biotechnologies, Madison, WI, USA) according to the manufacturer's instructions. A paired set of sample RNAs that were not treated with Ribo-Zero were ethanol precipitated and suspended in $3 \mu \mathrm{l}$ of QuickExtract RNA Extraction Solution to assess rRNA contamination in subsequent sequencing. Purified viral RNAs from corresponding VICs were included, but not subjected to the Ribo-Zero protocol as these samples were hypothesized to contain more influenza RNA. RiboZero-treated and untreated samples were then used for unbiased whole-genome linear amplification using the MessageBOOSTER $^{\mathrm{TM}}$ cDNA Synthesis Kit (Epicentre 
Biotechnologies) as per the manufacturer's instructions with the modification of using a T7 random hexamer primer (5'-AAT TGT AAT ACG ACT CAC TAT AGG G(N: 25252525)(N) (N)V(N) N-3', where $N$ includes $A, C, G$, $\mathrm{T}$, and $\mathrm{V}$ includes $\mathrm{A}, \mathrm{C}, \mathrm{G})$ for the first-strand cDNA synthesis. The oligo was ordered as 'Customized Hand-Mix' for mixing of all ' $N$ ' and ' $V$ ' bases at equal ratio. Secondstrand synthesis was achieved by the addition of $100 \mathrm{ng}$ of random hexamers, dNTPs and Klenow Fragment followed by incubation at $37^{\circ} \mathrm{C}$ for $30 \mathrm{~min}$ and heat inactivation. The cDNAs were then purified using the DNA Clean \& Concentrator ${ }^{\mathrm{TM}}-5$ (Zymo Research, Irvine, CA, USA) and used for library preparation using the Nexter$\mathrm{a}^{\mathrm{TM}}$ DNA Sample Prep Kit, Nextera ${ }^{\mathrm{TM}}$ PCR Enzyme and Nextera $^{\mathrm{TM}}$ Bar Codes (Epicentre Biotechnologies) according to the manufacturer's instructions.

\section{Illumina data analysis}

Sixteen samples of 105 paired-end sequences were produced using two lanes of the Illumina GAIIx. Cluster identification and filtering, base calling and uncertainty assessment were all performed in real time using scs 2.8/ RTA 1.8 (Illumina, Inc., San Diego, CA, USA) software with the default parameter settings. After initial formatting of the sequence data using BCLCONVERTER-1.7.1 (Illumina, Inc.), the sequences were de-multiplexed using CASAVA-1.7.0 (Illumina, Inc.). For each of the 16 samples, data passing the initial Illumina data quality filter were converted to FASTQ format using CASAva-1.7.0. As a final step prior to bioinformatics analysis, adaptor, T7, transposon and other technical sequences appended to the ends of the insert fragments were trimmed using Cutadapt (Martin, 2011).

Because the sequences originated from biologically derived samples, the bioinformatics analysis began by classifying each sequence read using BWA (Li and Durbin, 2009) and the following reference genomes: (i) Pandemic H1N1 (A/California/04/2009(H1N1)); (ii) Swine (NCBI genome assembly Sscrofa10.2); (iii) Swine $18 \mathrm{~S}$ rRNA (NR_002170.3); and (iv) Virus, Bacteria and Fungi. These genomes were derived from fludb.org and NCBI nucleotide BLAST+ databases (Camacho et al., 2009). Alignment of the trimmed sequences to the reference genomes was performed in a 'stringent' manner, only allowing up to five mismatches total with at most one mismatch in the first 32 bases.

\section{Phylogenetic analysis of sanger data}

To trace the phylogenetic evolution of the 2011 swine pH1N1 isolate, all available influenza sequence data from 2009 to 2011 were downloaded from fludb.org and for- matted into a BLAST+ database (Camacho et al., 2009). Each of the sixteen segments derived from VIC10253 and VIC10254 sequencing were blasted against this database without restricting the number of hits returned. Approximately 25 of the hits that were evenly spaced between the best hit matching the pH1N1 feral swine sequences and the A/California/04/2009(H1N1) hit were selected, and a phylogenetic tree was generated using Clustal w (Thompson et al., 1994) and the molecular evolutionary genetics analysis version 5 (MEgA5; Tamura et al., 2011). The phylogenetic evolution of the VIC10254 sequences was next assessed with respect to a set of related influenza strains. The best matching isolates were identified by blasting each of the 2011 feral swine pH1N1 segments against the sequence-identity search tool at NCBI. Finally, phylogenetic trees were generated using the close-neighbour-joining statistical method employing the Tamura-Nei nucleotide substitution model (Tamura and Nei, 1993) and 500 bootstrap replicate options.

Single nucleotide polymorphisms (SNP) and quasispecies analysis of illumina sequences

The Illumina reads for VIC10254 were used for SNP and quasispecies analyses with respect to a selected set of influenza strain references: swine $\mathrm{pH} 1 \mathrm{~N} 1$ with the highest identity by BLAST, swine $\mathrm{pH} 1 \mathrm{~N} 1$ prototype $\mathrm{A} /$ swine/ Alberta/OTH-33-8/2009 and human $\mathrm{pH} 1 \mathrm{~N} 1$ prototype A/ California/04/2009. SNPs were determined using the default procedure of SAMtools (Li et al., 2009). To be reported as a SNP, the quality of a candidate SNP base must be greater than 13 on a $0-40$ Phred score scale. Quasispecies were analysed using all gene segments of both VIC10254 and NS10254 using A/California/04/2009 as a reference sequence. Quasispecies are indicated by positions where there are two bases in the reads that pass the SNP quality threshold and are different from the reference base.

\section{Results}

\section{RT-qPCR and virus isolation}

Two feral swine nasal swab samples, NS10253 and NS10254, were identified as positive for the SIV matrix gene and the 2009 pandemic N1 by RT-qPCR.

Virus isolation results were confirmed by RT-qPCR using cell culture supernatants. Virus isolates obtained from the samples NS10253 and NS10254 were named A/ swine/Texas/A01104003/2011 and A/swine/Texas/A011040 04/2011, respectively, but for practical purposes during sequencing, virus isolation culture (VIC) samples were named VIC10253 and VIC10254, respectively. 
Table 1. Genbank accession number for all eight segments of feral swine isolates

\begin{tabular}{|c|c|c|c|c|c|c|c|c|}
\hline \multirow[b]{2}{*}{ Virus isolate } & \multicolumn{8}{|c|}{ Influenza RNA segments } \\
\hline & PB2 & PB1 & PA & $\mathrm{HA}$ & $\mathrm{NP}$ & NA & M & NS \\
\hline A/swine/Texas/A01104003/2011 & JN673247 & JN673248 & JN673249 & JN673250 & JN673251 & JN673252 & JN673253 & JN673254 \\
\hline A/swine/Texas/A01 104004/2011 & JN673255 & JN673256 & JN673257 & JN673258 & JN673259 & JN673260 & JN673261 & JN673262 \\
\hline
\end{tabular}

\section{Sanger sequencing}

Sequences for all eight segments of the isolates $\mathrm{A} /$ swine/ Texas/A01104003/2011 and A/swine/Texas/A01104004/ 2011 were successfully obtained and were submitted to GenBank as indicated in Table 1. Sanger sequences of the two isolates were compared by BLAST pairwise alignment. Three nucleotide substitutions in three segments were observed: A221G (silent) in PB1, T194C (S65F) in $\mathrm{PA}$ and $\mathrm{A} 9 \mathrm{C}$ (silent) in HA (where the first letter denotes the base in VIC10253, the number denotes the base position and the second letter denotes the base in VIC10254, followed by the amino acid change). The highest sequence identity by BLAST was observed with human pH1N1 sequences for all segments, which may suggest that this isolate was derived from human transmission.

\section{Illumina next generation sequencing}

The results for the analysed sequence classification and summary statistics of the alignment, including the total count of reads that could be mapped to each of the references, are given in Table 2; however, mapping was not exclusive, and a single read can appear in the count of multiple columns. Thus, the unknown column, defined as the reads post-filter column minus the sum of the other (non-coverage) columns, is a rough estimate of the number of reads whose identity could not be discerned and may be negative. A further breakdown of the sequencing coverage for each $\mathrm{pH} 1 \mathrm{~N} 1$ segment is given in Table 3. In contrast to Table 2, which gives raw read counts, the coverage in Table 3 is defined as the number of bases aligned to a $\mathrm{pH} 1 \mathrm{~N} 1$ segment divided by the number of bases comprising the segment itself. The total number of fragments passing and their distribution between the multiplexed samples were consistent with a successful GAIIx run. However, the total number of sequences that could be classified as pH1N1 was low for NS10253 and VIC10253. The results from nasal swab samples, which contained environmental nucleic acids, were reduced as expected because the overall contribution of influenza genetic material is relatively small. In all samples, swine and unknowns appear to be the dominant contributors of genetic material, although bacteria and fungi were also substantial contributors to the genetic material sequenced.

Because of the relatively high coverage, the guided assembly was able to produce nearly full-length consensus assemblies of all segments for VIC10253 and VIC10254.

Table 2. Illumina next generation sequencing analysis. Sequence classification and summary statistics

\begin{tabular}{|c|c|c|c|c|c|c|c|c|c|}
\hline \multirow[b]{2}{*}{ Sample ${ }^{a}$} & \multirow[b]{2}{*}{ Reads post-filter } & \multicolumn{8}{|c|}{ Reads mapped to: } \\
\hline & & Reference $^{\mathrm{b}}$ & Coverage $^{c}$ & Swine genome & 18S rRNA & Viral $^{\mathrm{d}}$ & Bacterial & Fungal & Unknowns \\
\hline$N S 10253+R$ & 4141724 & 15 & 1 & 1798081 & 1136 & 4454 & 249281 & 9914 & 2079979 \\
\hline NS10253-R & 5227520 & 31 & 4 & 2066712 & 3450 & 7720 & 398510 & 41197 & 2713350 \\
\hline VIC 10253-R & 3326208 & 28256 & 91 & 2849224 & 1729430 & 6314 & 74618 & 36340 & 331456 \\
\hline NS10254+R & 7346365 & 4035 & 58 & 7976911 & 1592 & 10629 & 65657 & 14541 & -725408 \\
\hline NS10254-R & 7365714 & 7432 & 53 & 7286625 & 9393 & 6011 & 117041 & 45116 & -96511 \\
\hline NS10254-10+R & 5437316 & 1739 & 47 & 3944613 & 1361 & 11335 & 386570 & 21049 & 1072010 \\
\hline NS10254-10-R & 6910107 & 2138 & 58 & 2566690 & 13693 & 12050 & 1459790 & 128018 & 2741421 \\
\hline NS10254-100+R & 2588569 & 141 & 7 & 515780 & 607 & 52992 & 335619 & 69688 & 1614349 \\
\hline NS10254-100-R & 6499963 & 481 & 27 & 2152934 & 14920 & 14540 & 1444692 & 115178 & 2772138 \\
\hline VIC 10254-R & 6166530 & 330135 & 96 & 4114685 & 1990988 & 53677 & 203834 & 67601 & 1396598 \\
\hline$N S P-R$ & 11024308 & 30 & 1 & 3645245 & 17398 & 19328 & 2849724 & 172818 & 4337163 \\
\hline
\end{tabular}

The per cent of the Pandemic H1N1 reference that was covered by aligned reads is given in the column coverage.

${ }^{a}(+) R$ denotes Ribo-Zero treatment to reduce ribosomal RNA. NSP denotes negative swab suspension pool.

${ }^{b}$ A/California/04/2009(H1N1).

'Per cent of reference genome coverage.

${ }^{d}$ Viral genomes exclusive of Influenza and denoted genomes. 
Table 3. Depth of coverage for each pH1N1 viral RNA segment

\begin{tabular}{|c|c|c|c|c|c|c|c|c|c|}
\hline \multirow[b]{2}{*}{ Segment } & \multicolumn{9}{|c|}{ Segment coverage } \\
\hline & VIC 10253-R & NS10254+R & NS10254-R & NS10254-10+R & NS10254-10-R & NS10254-100+R & NS10254-100-R & VIC 10254-R & $N S P-R$ \\
\hline PB1 & 392 & 46 & 90 & 19 & 26 & 1 & 6 & 4224 & 0 \\
\hline PA & 244 & 35 & 57 & 17 & 16 & 1 & 4 & 2807 & 0 \\
\hline NS & 186 & 39 & 46 & 15 & 13 & 1 & 3 & 2515 & 0 \\
\hline PB2 & 176 & 18 & 47 & 9 & 13 & 2 & 3 & 2033 & 0 \\
\hline NP & 142 & 21 & 48 & 8 & 13 & 1 & 3 & 1869 & 0 \\
\hline $\mathrm{HA}$ & 118 & 27 & 42 & 11 & 13 & 1 & 2 & 1566 & 0 \\
\hline NA & 105 & 18 & 38 & 7 & 11 & 1 & 2 & 1241 & 0 \\
\hline $\mathrm{M}$ & 59 & 15 & 13 & 7 & 4 & 0 & 1 & 727 & 0 \\
\hline
\end{tabular}

Coverage is defined as the number bases aligned to a pH1N1 segment divided by the number of bases comprising the segment itself.

There were definite end effects that caused the coverage at the ends of the segments to be reduced compared to the middle sections of the segments. Where not limited by end effects, the guided assembly and the VIC10254 aligned reads were used to analyse the SNPs using a selected set of reference sequences to demonstrate the sequence divergence. Analysis of all eight gene segments using the A/California/04/2009 reference identified the largest number of SNPs in the HA segment $(n=20)$ and the lowest number of SNPs in the NS segment $(n=3)$. Quasispecies analysis did not identify any base variations in VIC10254. Two base variations were identified in NS10254 PB1 at nucleotide positions 1398 and 1791. Although the total coverage of these positions is low, all reads passed the established base and SNP calling quality thresholds. The low number of sequence variants observed may be a result of the overall influenza sequence coverage for the analysed samples and improvements in library preparation methods, or the use of influenza sequence enrichment methods may enable better identification of quasispecies as has been recently described (Kampmann et al., 2011).

\section{Phylogenetic analysis}

Phylogenetic analysis of Sanger full-genome sequences for all eight segments of VIC10253 and VIC10254 indicated that all segments are grouped within the pH1N1 lineage. Further analysis of the VIC10254 HA segment using a selected set of representative sequences is shown in Fig. 1. The phylogenetic results placed VIC10253 and VIC10254 into the human pH1N1 cluster for all segments analysed; swine reassortant segments were also correctly clustered.

\section{Discussion}

Feral swine currently play a role in the spread and emergence of many diseases, some of which have significant implications for human health. They act as reservoirs for swine influenza $\mathrm{H} 1 \mathrm{~N} 1$ and $\mathrm{H} 3 \mathrm{~N} 2$, which are endemic in domestic swine populations worldwide. We report the identification of the first $\mathrm{pH} 1 \mathrm{~N} 1$ from feral swine in the United States.

The pH1N1 (2009) virus is a reassortant virus containing a unique combination of gene segments from SIVs in North America and Eurasia and from avian influenza viruses in North America (Garten et al., 2009). Bayesian molecular-clock analysis of $\mathrm{pH} 1 \mathrm{~N} 1$ viruses has suggested that the duration of unsampled diversity for each gene segment had means that ranged from 9.24 to 17.15 years, suggesting that the $\mathrm{pH} 1 \mathrm{~N} 1$ progenitor virus may have been circulating undetected for nearly a decade (Smith et al., 2009, Welsh et al., 2010).

The maintenance of pH1N1 within feral and domestic pig populations provides further reassortment opportunities with endemic SIVs. Thus far, pH1N1 and SIV reassortments have generated novel H1N1 (Vijaykrishna et al., 2010; Ducatez et al., 2011; Kitikoon et al., 2011; Starick et al., 2011; Zhu et al., 2011), H1N2 (Ducatez et al., 2011; Howard et al., 2011; Moreno et al., 2011) and H3N2 (Ducatez et al., 2011; Tremblay et al., 2011) viruses. All but two of these reassortant strains contained the $\mathrm{M}$ gene segment of $\mathrm{pH} 1 \mathrm{~N} 1$, which may suggest a selective advantage to viruses containing this segment (Chou et al., 2011; Ducatez et al., 2011). Our results agree with previous reports demonstrating that most reassortant strains contain $\mathrm{pH} 1 \mathrm{~N} 1-$ derived $\mathrm{M}$ segments. The high transmissibility of the $\mathrm{pH} 1 \mathrm{~N} 1 \mathrm{M}$ segment warrants surveillance of this virus in the pig population to monitor future viral evolution, the extent of reassortment and the impact of new strains on human health.

While research on viral reassortment has not been conducted in feral swine, feral swine are the same species as domestic swine, sharing the same physiology and similar susceptibilities to disease. At the same time, feral swine are free roaming and thus have behaviour and habitat 


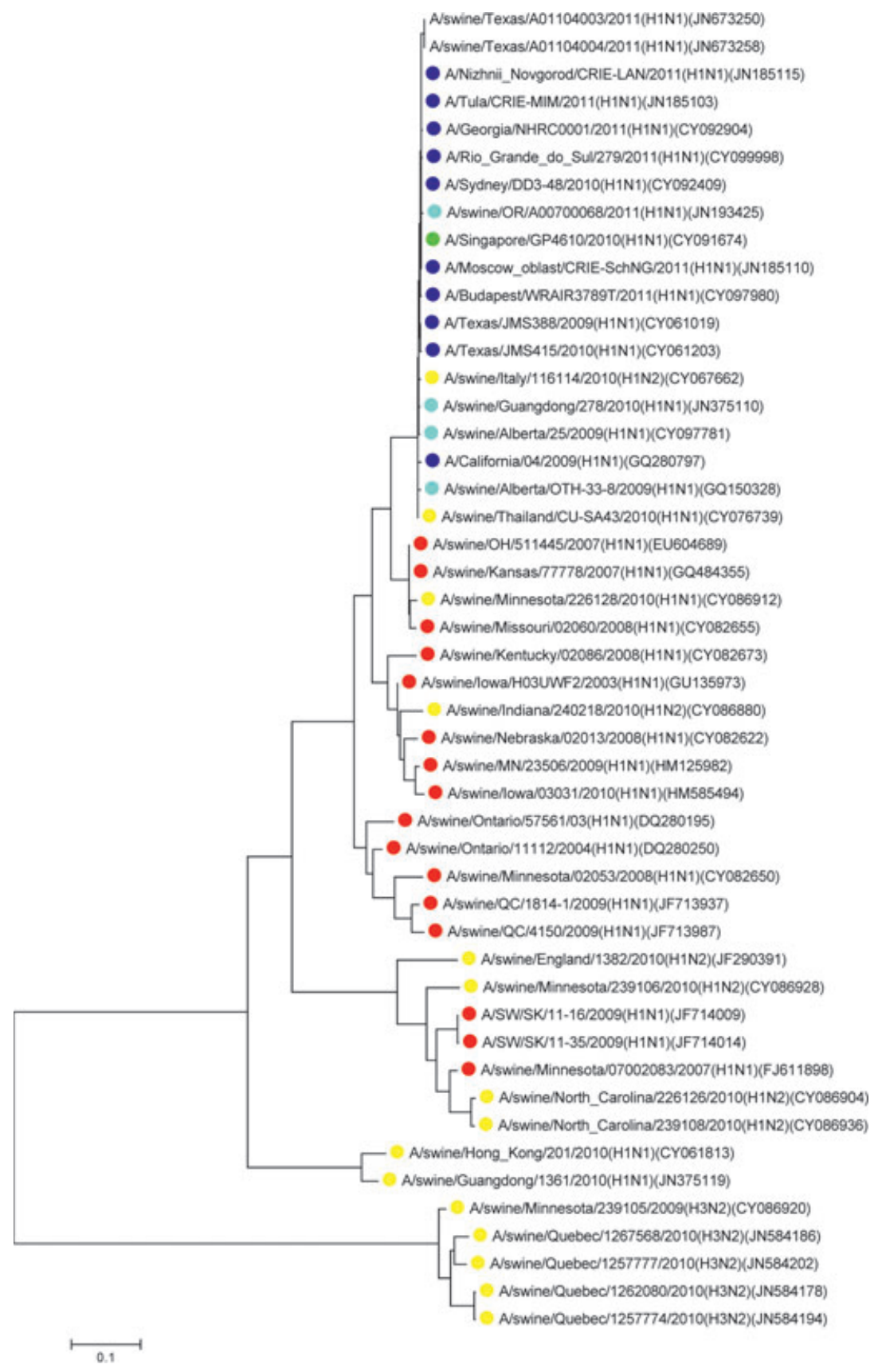

Fig. 1. Molecular phylogenetic analysis of the HA segment by the maximum likelihood method. Viruses selected for analysis were the highest identity by BLAST (green), swine pH1N1 (cyan), human pH1N1 (blue), swine reassortant strains with pH1N1(yellow) and classical swine H1N1 (red). The evolutionary history for each segment was inferred using the maximum likelihood method based on the Tamura-Nei model. The tree is drawn to scale, with branch lengths measured in the number of substitutions per site. Evolutionary analyses were conducted in MEGA5.

preferences that bring them into contact with wild waterfowl, domestic swine, poultry and humans, making them potential vectors for the transfer of influenza virus between and among species. Endemic strains of SIV have been circulating in the US swine population for over 75 years (Webby et al., 2004; Hall et al., 2008).
The current NWDP surveillance program includes SIV testing. Samples are collected in 25 of the 38 states currently known to have feral swine. Nasal swabs are collected in targeted counties and tested at the National Animal Health Laboratory Network (NAHLN) laboratories. As of 30 September 2011, the NWDP had sampled and 
submitted 1895 nasal swabs and 2501 serum samples collected from across the United States. Of the serum samples, $256(10.2 \%)$ tested positive for Type A influenza antibodies. Of the nasal swabs, 13 tested positive by RT-qPCR, but the virus was not recovered from 11 of the 13 samples. Two of the RT-qPCR positive samples yielded isolated virus from tissue culture, and the virus was identified as $\mathrm{pH} 1 \mathrm{~N} 1$; these isolates were the subject of this report.

Traditional Sanger and next generation Illumina GAIIx sequencing methods were used to obtain full-genome sequences for the two viral isolates. Both methods provided equivalent sequencing data and phylogenetic results, indicating that all segments of the new virus were derived from the $\mathrm{pH} 1 \mathrm{~N} 1$ lineage.

The next generation sequencing results provided feasibility data supporting the use of a simple unbiased wholegenome linear amplification library preparation method and sample pooling of up to eight samples per lane. Analysis of Illumina pH1N1 sequence reads indicated that the PB2 segment contained the highest sequence coverage for all samples, while the $M$ segment contained the lowest coverage for all samples; because a limited number of samples $(n=2)$ were evaluated, the reason for this observation is unclear. Direct diagnostic biological samples also produced high-quality sequence reads that resulted in $>50 \%$ coverage of the influenza genome. Furthermore, even a 100 -fold dilution ( $\sim \mathrm{M}$ Ct 26$)$ still enabled the detection of influenza sequences, indicating that direct diagnostic samples containing diverse viral titres may be analysed in the context of metagenomics to determine the pathogen of interest for differential diagnosis.

In addition to the NGS technical methods described, a bioinformatics pipeline was also established for the streamlined analysis of these data. These tools provide a process to enable NGS applications in pathogen-related research and diagnostics including syndromic disease diagnostics and pathogen discovery, disease surveillance and outbreak detection, and other critical diagnostic applications.

We report the detection of a $\mathrm{pH} 1 \mathrm{~N} 1$ virus from U.S. feral swine populations in which all genes are derived from the pH1N1 (2009) virus. The source of the viruses isolated from feral swine could not be established. The highest sequence identity by BLAST was observed with human pH1N1 sequences for all segments, which may suggest that these isolates were derived from human viruses transmitted to swine. However, the progenitor virus of $\mathrm{pH} 1 \mathrm{~N} 1$ is proposed to have originated in pigs, even though to date, the virus has not been detected in pigs before its emergence in humans (Welsh et al., 2010). It is therefore possible that the viruses could have originated from domestic swine. Feral swine are abundant in Texas and have become even more widely distributed in recent years, resulting in an increased risk for the transmission of influenza between feral swine and commercial and transitional swine (captive feral swine or swine with a reasonable opportunity of being exposed to feral swine; Hutton et al., 2006). This association may also present a risk for the transmission of other serious zoonotic diseases, such as brucellosis and leptospirosis, to humans that are either directly or indirectly exposed to feral swine (Meng et al., 2009).

Swine influenza virus can be transmitted via direct contact and droplets, and thus, it may be spread from commercial or transitional swine to feral swine in the vicinity of commercial swine barns or from feral swine to commercial and transitional swine. Subsequent spread among feral swine could occur where feral swine densities allow for a sufficient contact rate. Biosecurity at most commercial swine barns precludes direct contact between feral and commercial swine, but the biosecurity for transitional swine is limited or non-existent and direct contact is possible. Additional epidemiological studies are required to understand the potential transmission risk of $\mathrm{pH} 1 \mathrm{~N} 1$ and other reassortant SIV among feral swine; the role of feral swine as a reservoir for influenza; and the potential mechanisms by which influenza can be transmitted from feral swine to commercial and managed feral swine, from commercial swine to feral swine and ultimately from swine to human populations. Our study also highlights the importance of educating hunters and others who encounter feral swine on the risk of acquiring zoonotic diseases, such as SIV, and on methods to reduce this risk. Understanding the viral shift and drift in influenza viruses in swine populations is important for understanding the potential risks of transmission to other species, including humans.

\section{Acknowledgements}

We are highly grateful to Dr. Scott Kuersten of Epicentre Biotechnologies (An Illumina Company) for valuable technical assistance in target amplification and Illumina DNA library methods and donations of Epicentre reagents. The authors thank Vanessa Lucas, Feng Sun and Shirley Byrne for their excellent technical assistance.

\section{References}

Animal and Plant Inspection Services, 2012: Veterinary services national surveillance plan for swine influenza virus in pigs. Available at: http://www.aphis.usda.gov/animal_health/animal_dis_spec/swine/siv_surv_manual.shtm (accessed on 15 January 2012).

Camacho, C., G. Coulouris, V. Avagyan, N. Ma, J. Papadopoulos, K. Bealer, and T. L. Madden, 2009: BLAST+: architecture and applications. BMC Bioinformatics 10, 421. 
Chan, C. H., K. L. Lin, Y. Chan, Y. L. Wang, Y. T. Chi, H. L. Tu, H. K. Shieh, and W. T. Liu, 2006: Amplification of the entire genome of influenza A virus $\mathrm{H} 1 \mathrm{~N} 1$ and $\mathrm{H} 3 \mathrm{~N} 2$ subtypes by reverse-transcription polymerase chain reaction. J. Virol. Methods 136, 38-43.

Chou, Y. Y., R. A. Albrecht, N. Pica, A. C. Lowen, J. A. Richt, A. Garcia-Sastre, P. Palese, and R. Hai, 2011: The M segment of the 2009 new pandemic H1N1 influenza virus is critical for its high transmission efficiency in the guinea pig model. J. Virol. 85, 11235-11241.

Ciacci-Zanella, J. R., A. L. Vincent, J. R. Prickett, S. M. Zimmerman, and J. J. Zimmerman, 2010: Detection of anti-influenza A nucleoprotein antibodies in pigs using a commercial influenza epitope-blocking enzyme-linked immunosorbent assay developed for avian species. J. Vet. Diagn. Invest. 22, 3-9.

Clavijo, A., D. B. Tresnan, R. Jolie, and E. M. Zhou, 2002: Comparison of embryonated chicken eggs with MDCK cell culture for the isolation of swine influenza virus. Can. J. Vet. Res. 66, 117-121.

Dawood, F. S., S. Jain, L. Finelli, M. W. Shaw, S. Lindstrom, R. J. Garten, L. V. Gubareva, X. Xu, C. B. Bridges, and T. M. Uyeki, 2009: Emergence of a novel swine-origin influenza A (H1N1) virus in humans. N. Engl. J. Med. 360, 2605-2615.

Ducatez, M. F., B. Hause, E. Stigger-Rosser, D. Darnell, C. Corzo, K. Juleen, R. Simonson, C. Brockwell-Staats, A. Rubrum, D. Wang, A. Webb, J. C. Crumpton, J. Lowe, M. Gramer, and R. J. Webby, 2011: Multiple reassortment between pandemic (H1N1) 2009 and endemic influenza viruses in pigs, United States. Emerg. Infect. Dis. 17, 1624 1629.

Forgie, S. E., J. Keenliside, C. Wilkinson, R. Webby, P. Lu, O. Sorensen, K. Fonseca, S. Barman, A. Rubrum, E. Stigger, T. J. Marrie, F. Marshall, D. W. Spady, J. Hu, M. Loeb, M. L. Russell, and L. A. Babiuk, 2011: Swine outbreak of pandemic influenza A virus on a Canadian research farm supports human-to-swine transmission. Clin. Infect. Dis. 52, 1018.

Garten, R. J., C. T. Davis, C. A. Russell, B. Shu, S. Lindstrom, A. Balish, W. M. Sessions, X. Xu, E. Skepner, V. Deyde, M. Okomo-Adhiambo, L. Gubareva, J. Barnes, C. B. Smith, S. L. Emery, M. J. Hillman, P. Rivailler, J. Smagala, M. De Graaf, D. F. Burke, R. A. Fouchier, C. Pappas, C. M. Alpuche-Aranda, H. Lo' pez-Gatell, H. Olivera, I. Lo'pez, C. A. Myers, D. Faix, P. J. Blair, C. Yu, K. M. Keene, P. D. Dotson Jr, D. Boxrud, A. R. Sambol, S. H. Abid, K. St. George, T. Bannerman, A. L. Moore, D. J. Stinger, P. Blevins, G. J. Demmler-Harrison, M. Ginsberg, P. Kriner, S. Waterman, S. Smole, H. F. Guevara, E. A. Belongia, P. A. Clark, S. T. Beatrice, R. Donis, J. Katz, L. Finelli, C. B. Bridges, M. Shaw, D. B. Jernigan, T. M. Uyeki, D. J. Smith, A. I. Klimov, and N. J. Cox, 2009: Antigenic and genetic characteristics of swine-origin 2009 (H1N1) influenza viruses circulating in humans. Science 325, 197-201.
Hall, J. S., R. B. Minnis, T. A. Campbell, S. Barras, R. W. Deyoung, K. Pabilonia, M. L. Avery, H. Sullivan, L. Clark, and R. G. McLean, 2008: Influenza exposure in United States feral swine populations. J. Wildl. Dis. 44, 362-368.

Howard, W. A., S. C. Essen, B. W. Strugnell, C. Russell, L. Barass, S. M. Reid, and I. H. Brown, 2011: Reassortant Pandemic (H1N1) 2009 virus in pigs, United Kingdom. Emerg. Infect. Dis. 17, 1049-1052.

Hutton, T., T. DeLiberto, and S. Owen, 2006: Disease Risk Associated with Increasing Feral Swine Numbers and Distribution in the United States. APHIS-Wildlife Services. Available at: http://www.michigan.gov/documents/mda/ Hutton_Pig_Paper_218759_7.pdf (accessed on 20 August 2012).

Kampmann, M. L., S. L. Fordyce, M. C. Avila-Arcos, M. Rasmussen, E. Willerslev, L. P. Nielsen, and M. T. Gilbert, 2011: A simple method for the parallel deep sequencing of full influenza A genomes. J. Virol. Methods 178, 243-248.

Kitikoon, P., D. Sreta, S. N. Na Ayudhya, M. Wongphatcharachai, J. Lapkuntod, D. Prakairungnamthip, N. Bunpapong, S. Suradhat, R. Thanawongnuwech, and A. Amonsin, 2011: Brief report: molecular characterization of a novel reassorted pandemic H1N1 2009 in Thai pigs. Virus Genes 43, 1-5.

Li, H., and R. Durbin, 2009: Fast and accurate short read alignment with Burrows-Wheeler transform. Bioinformatics $25,1754-1760$.

Li, H., B. Handsaker, A. Wysoker, T. Fennell, J. Ruan, N. Homer, G. Marth, G. Abecasis, and R. Durbin, 2009: The Sequence Alignment/Map format and SAMtools. Bioinformatics 25, 2078-2079.

Martin, M., 2011: Cutadapt removes adapter sequences from high-throughput sequencing reads. Technical Notes, 2011; EMBnet.journal 17.1. Available at: http://journal.embnet.org/ index.php/embnetjournal/article/view/200/458 (accessed on 2 February 2012)

Meng, X. J., D. S. Lindsay, and N. Sriranganathan, 2009: Wild boars as sources for infectious diseases in livestock and humans. Philos. Trans. R. Soc. Lond. B Biol. Sci. 364, 26972707.

Moreno, A., T. L. Di, S. Faccini, G. Vaccari, D. Nigrelli, M. B. Boniotti, E. Falcone, A. Boni, C. Chiapponi, E. Sozzi, and P. Cordioli, 2011: Novel H1N2 swine influenza reassortant strain in pigs derived from the pandemic H1N1/2009 virus. Vet. Microbiol. 149, 472-477.

NCBI Influenza Virus Sequence Database, 2012. Available at: http://www.ncbi.nlm.nih.gov/genomes/FLU/FLU.html (accessed on 13 January 2012).

Nfon, C. K., Y. Berhane, T. Hisanaga, S. Zhang, K. Handel, H. Kehler, O. Labrecque, N. S. Lewis, A. L. Vincent, J. Copps, S. Alexandersen, and J. Pasick, 2011: Characterization of H1N1 swine influenza viruses circulating in Canadian pigs in 2009. J. Virol. 85, 8667-8679.

Njabo, K. Y., T. L. Fuller, A. Chasar, J. P. Pollinger, G. Cattoli, C. Terregino, I. Monne, J. M. Reynes, R. Njouom, and T. B. 
Smith, 2012: Pandemic A/H1N1/2009 influenza virus in Swine, Cameroon, 2010. Vet. Microbiol. 156, 189-192.

Pedersen, K., S. N. Bevins, B. S. Schmit, M. W. Lutman, M. P. Milleson, C. T. Turnage, T. T. Bigelow, and T. J. DeLiberto, 2012: Apparent prevalence of swine brucellosis in feral swine in the United States. Human-Wildlife Interact. 6, 38-47.

Pimental, D., 2007: Environmental and Economic Cost of Vertebrate Species Invasions Into the United States. USDA National Wildlife Research Center Symposia, U.S. Department of Agriculture. Available at: http://www.aphis.usda.gov/wildlife_damage/nwrc/symposia/invasive_symposium/ nwrc_TOC_index.shtml (accessed on 10 January 2012)

Saliki, J. T., S. J. Rodgers, and G. Eskew, 1998: Serosurvey of selected viral and bacterial diseases in wild swine from Oklahoma. J. Wildl. Dis. 34, 834-838.

Smith, G. J., D. Vijaykrishna, J. Bahl, S. J. Lycett, M. Worobey, O. G. Pybus, S. K. Ma, C. L. Cheung, J. Raghwani, S. Bhatt, J. S. Peiris, Y. Guan, and A. Rambaut, 2009: Origins and evolutionary genomics of the 2009 swine-origin H1N1 influenza A epidemic. Nature 459, 1122-1125.

Starick, E., E. Lange, S. Fereidouni, C. Bunzenthal, R. Hoveler, A. Kuczka, B. E. grosse, H. P. Hamann, I. Klingelhofer, D. Steinhauer, T. Vahlenkamp, M. Beer, and T. Harder, 2011: Reassorted pandemic (H1N1) 2009 influenza A virus discovered from pigs in Germany. J. Gen. Virol. 92, 1184-1188.

Swafford, S. R., B. B. Schmit, K. Pedersen, M. W. Lutman, and T. J. DeLiberto. 2009. Classical Swine Fever Surveillance in feral swine. In: Boulanger, J. R. (ed.) Proceedings of the 13th Wildlife Damage Management Conference.

Tamura, K., and M. Nei, 1993: Estimation of the number of nucleotide substitutions in the control region of mitochondrial DNA in humans and chimpanzees. Mol. Biol. Evol. 10, 512-526.

Tamura, K., D. Peterson, N. Peterson, G. Stecher, M. Nei, and S. Kumar, 2011: MEGA5: molecular evolutionary genetics analysis using maximum likelihood, evolutionary distance, and maximum parsimony methods. Mol. Biol. Evol. 28, 2731-2739.
Thompson, J. D., D. G. Higgins, and T. J. Gibson, 1994: CLUSTAL W: improving the sensitivity of progressive multiple sequence alignment through sequence weighting, position-specific gap penalties and weight matrix choice. Nucleic Acids Res. 22, 4673-4680.

Tremblay, D., V. Allard, J. F. Doyon, C. Bellehumeur, J. G. Spearman, J. Harel, and C. A. Gagnon, 2011: Emergence of a new swine H3N2 and pandemic (H1N1) 2009 influenza A virus reassortant in two Canadian animal populations, mink and swine. J. Clin. Microbiol. 49, 4386-4390.

Vijaykrishna, D., L. L. Poon, H. C. Zhu, S. K. Ma, O. T. Li, C. L. Cheung, G. J. Smith, J. S. Peiris, and Y. Guan, 2010: Reassortment of pandemic H1N1/2009 influenza A virus in swine. Science 328, 1529.

Webby, R. J., K. Rossow, G. Erickson, Y. Sims, and R. Webster, 2004: Multiple lineages of antigenically and genetically diverse influenza A virus co-circulate in the United States swine population. Virus Res. 103, 67-73.

Welsh, M. D., P. M. Baird, M. P. Guelbenzu-Gonzalo, A. Hanna, S. M. Reid, S. Essen, C. Russell, S. Thomas, L. Barrass, F. McNeilly, J. McKillen, D. Todd, V. Harkin, S. McDowell, B. Choudhury, R. M. Irvine, J. Borobia, J. Grant, and I. H. Brown, 2010: Initial incursion of pandemic (H1N1) 2009 influenza A virus into European pigs. Vet. Rec. 166, 642-645.

World Health Organization, Global Alert and Response (GAR), 2012: Pandemic (H1N1) 2009 - update 112. Available at: http://www.who.int/csr/don/2010_08_06/en/ index.html (accessed on 15 January 2012).

Wyckoff, A. C., S. E. Henke, T. A. Campbell, D. G. Hewitt, and K. C. VerCauteren, 2009: Feral swine contact with domestic swine: a serologic survey and assessment of potential for disease transmission. J. Wildl. Dis. 45, 422-429.

Zhu, H., B. Zhou, X. Fan, T. T. Lam, J. Wang, A. Chen, X. Chen, H. Chen, R. G. Webster, R. Webby, J. S. Peiris, D. K. Smith, and Y. Guan, 2011: Novel reassortment of Eurasian avian-like and pandemic/2009 influenza viruses in swine: infectious potential for humans. J. Virol. 85, 10432-10439. 